\title{
Estimation of the gravitational wave polarizations from a nontemplate search
}

\author{
Irene Di Palma* \\ Istituto Nazionale di Fisica Nucleare, Sezione di Roma, Italy \\ and Universitá di Roma La Sapienza, I-00185 Roma, Italy \\ Marco Drago ${ }^{\dagger}$ \\ Albert-Einstein-Institut, Max-Planck-Institut für Gravitationsphysik, \\ Callinstrasse 38, 30167 Hannover, Germany \\ and Leibniz Universität Hannover, Hannover, Germany
}

(Received 7 November 2016; published 18 January 2018)

\begin{abstract}
Gravitational wave astronomy is just beginning, after the recent success of the four direct detections of binary black hole $(\mathrm{BBH})$ mergers and the first observation from a binary neutron star inspiral, with the expectation of many more events to come. Given the possibility to detect waves from not exactly modeled astrophysical processes, it is fundamental to be ready to calculate the polarization waveforms in the case of searches using nontemplate algorithms. In such a case, the waveform polarizations are the only quantities that contain direct information about the generating process. We present the performance of a new valuable tool to estimate the inverse solution of gravitational wave transient signals, starting from the analysis of the signal properties of a nontemplate algorithm that is open to a wider class of gravitational signals not covered by template algorithms. We highlight the contributions to the wave polarization associated with the detector response, the sky localization, and the polarization angle of the source. In this paper we present the performances of such a method and its implications by using two main classes of transient signals, resembling the limiting case for most simple and complicated morphologies. The performances are encouraging for the tested waveforms: the correlation between the original and the reconstructed waveforms spans from better than $80 \%$ for simple morphologies to better than $50 \%$ for complicated ones. For a nontemplate search these results can be considered satisfactory to reconstruct the astrophysical progenitor.
\end{abstract}

DOI: 10.1103/PhysRevD.97.023011

\section{INTRODUCTION}

Gravitational waves (GWs) were predicted by Einstein's theory of general relativity in 1916 [1,2]. GWs are dynamic strains in space-time that travel at the speed of light and are generated by the nonaxisymmetric acceleration of mass. The discovery of the binary pulsar system PSR B1913+16 by Hulse and Taylor [3] and subsequent observations of its energy loss by Taylor and Weisberg [4] demonstrated the indirect existence of gravitational waves. Such discoveries led to the identification of the importance of direct observations of gravitational waves to study relativistic systems and test general relativity.

The GW community developed a network of groundbased laser interferometers including the two LIGO and Virgo detectors $[5,6]$. LIGO built the pair of detectors in Hanford, Washington and Livingston, Louisiana, while Virgo built the one in Pisa, Italy. These three detectors performed joint scientific runs from 2007 to 2010, putting

\footnotetext{
*Irene.DiPalma@roma1.infn.it

†Marco.Drago@aei.mpg.de
}

interesting upper limits on the detections of GWs [7-10]. Also in Europe, the smaller GEO600 detector [11] has been running and keeping watch on the GW universe, especially while its larger siblings were down for upgrades. The construction of the second-generation interferometers $[12,13]$ led to the first observing run in September, 2015 for the Advanced LIGO detectors [14]. The Virgo detector has been upgraded to Advanced Virgo [15] and recently joined the scientific run with LIGO. Looking ahead, the KAGRA (Kamioka Gravitational Wave Detector) detector $[16,17]$ is under construction in an underground site at the Kamioka mine, in Japan. Recently, another interferometer has been approved with an Indian location [18].

On September 14, 2015, the two LIGO interferometers detected the merger of a binary black hole system for the first time [19-21]. The signal event, named GW150914 — which was simultaneously observed in the two LIGO observatories [21] and first noted in a low-latency analysis for generic gravitational wave transients $[22,23]$-matched the waveform predicted by general relativity of the inspiral and merger of a pair of back holes and the ringdown of the resulting single one $[24,25]$. Three other events were 
detected in December, 2015, January, 2017, and June, 2017, together with a candidate event in October, 2015; they also matched waveforms generated by two black holes orbiting around each other and merging into a single one [26-29]. Advanced Virgo became operational on August 1, 2017 to join the second scientific run of the Advanced LIGO detectors. The three-detector network identified gravitational waves from a binary black hole coalescence, GW170814, improving the sky localization of the source, and reducing the area of the $90 \%$ credible region from $1160 \mathrm{deg}^{2}$ using only the two LIGO detectors to $60 \mathrm{deg}^{2}$ using all three detectors [30]. On August 17, 2017 the Advanced LIGO and Advanced Virgo detectors made their first observation of a binary neutron star inspiral [31]. The combination of data from the three interferometers allowed the most precisely localized gravitational-wave signal yet and enabled an extensive electromagnetic follow-up campaign that identified a counterpart near the galaxy NGC 4993, consistent with the localization and distance deduced from gravitational wave data [32]. Using the association between the luminosity distance directly measured from the gravitational wave signal and the galaxy NGC 4993 it is possible also to infer the Hubble constant [33]. These results affirm the beginning of GW astronomy as well as provide unprecedented observational insights into the physics of binary black holes [34], the physics of binary neutron stars, and the beginning of multimessenger astronomy.

Future events might pose the problem of detecting signals that are not strictly modeled like these first detected systems. Already aware of this issue, the GW community has developed coherent data analysis techniques which do not require prior knowledge of the signal and are open to a wide possible set of waveform shapes. These procedures have been applied in past analyses, especially in burst searches $[7,35]$. We use the word burst to identify all the signals which have limited time duration (less than seconds) and also include astrophysical processes for which there is not a complete model of the expected gravitational wave. For this reason, in the burst searches we make no particular assumptions on the waveform. Such coherent methods $[36,37]$ combine data from multiple detectors and create a unique list of candidate events for the whole network. A well-known advantage of coherence is its utility in rejecting background noise glitches [38,39]. Glitch rejection is particularly important since it is the limiting factor in the sensitivity of current burst searches, where a confident detection of a gravitational wave burst depends critically on how many glitches pollute the background estimation.

A consistent difference between these methods and modeled search filter algorithms is that they do not directly estimate the two waveform polarizations; rather, they reconstruct the projections of the polarizations for the different detectors, as done in Refs. [23,40]. In this paper, we present a completely new algorithm that can serve as a follow-up tool to reconstruct the original waveform polarizations starting from the information given by a generic unmodeled pipeline. As an example, we show the results of the application of this new algorithm to coherent Waveburst, the same pipeline that made the first alert of the GW150914 signal [19]. In Sec. II we introduce the theoretical calculations behind this work, and how we calculate the signal polarizations starting from information about the detector response and the source localization. Section III describes our simulations, and our results presented in Sec. IV.

\section{INVERSE SOLUTION}

The projection of the gravitational wave polarizations on a single interferometer is described by the so-called antenna patterns, which define the relative interferometer sensitivity in different directions. Each detector is sensitive to a linear combination of the two polarizations and has a quadrupolar antenna pattern. In the notation of Ref. [41], we consider the interferometer's arms along the $x$ and $y$ axes, and hence a generic gravitational wave can be described by the two polarization components $\left(h_{+}(t), h_{\times}(t)\right)$ in the $x-y$ plane and rotated by the polarization angle $\psi$, while the arrival direction is given by the spherical coordinates $\theta$ and $\phi$ relative to the detector's axes. For convenience we use the same approach, shown in Ref. [42], to represent detectors as vectors. The projection on a network of interferometric detectors is defined by the vector of detector responses,

$$
\xi_{\Delta}(\mathbf{t})=\mathbf{F}_{+}(\theta, \phi, \psi) h_{+}(t)+\mathbf{F}_{\times}(\theta, \phi, \psi) h_{\times}(t),
$$

where each vector component refers to a specific detector $k$. The quantity $\left\{\xi_{\Delta}(t)\right\}_{k}=\xi\left(t+\Delta_{k}\right)$ takes into account the relative difference in arrival time between the detector location and the Earth's center as a reference location ( $\Delta_{k}$, depending on the $\theta, \phi$ coordinates), while $\mathbf{F}_{+, \times}$are the detector antenna patterns, which are related to the relative orientation of the detector arms with respect to the source direction and the wave polarization frame. Since the new tool we are presenting uses the outputs of a standard coherent GW transient pipeline, i.e., the source direction $(\theta, \phi, \psi)$ and the detector response vector $\left(\xi_{\Delta}(\mathbf{t})\right)$, we have to solve the system in Eq. (1) to compute the polarization patterns.

From the literature [43,44] it is known that it is not possible to distinguish among the two polarizations with only two detectors; for this reason, we consider the case of networks composed of $N>2$ detectors. In such a case, we have for each data sample a redundant number of equations with respect to the unknown variable. By introducing the scalar products

$$
\begin{aligned}
& \mathbf{F}_{+} \cdot \xi_{\Delta}(\mathbf{t})=\left|\mathbf{F}_{+}\right|^{2} h_{+}(t)+\mathbf{F}_{+} \cdot \mathbf{F}_{\times} h_{\times}(t), \\
& \mathbf{F}_{\times} \cdot \xi_{\Delta}(\mathbf{t})=\mathbf{F}_{\times} \cdot \mathbf{F}_{+} h_{+}(t)+\left|\mathbf{F}_{\times}\right|^{2} h_{\times}(t),
\end{aligned}
$$

we reduce the problem to two equations in two variables $\left(h_{+}(t), h_{\times}(t)\right)$. All the other quantities are given by the 
TABLE I. Sketch of the different cases presented in this paper to disentangle the various contribution of source errors. Each case uses reconstructed and/or injected quantities.

\begin{tabular}{lccc}
\hline \hline $\begin{array}{l}\text { Case } \\
\text { Color }\end{array}$ & $\begin{array}{c}\text { Detector } \\
\text { Response }\end{array}$ & $\begin{array}{c}\text { Sky } \\
\text { Localization }\end{array}$ & $\begin{array}{c}\text { Polarization } \\
\text { Angle }\end{array}$ \\
\hline (a) & reconstructed & injected & injected \\
(b) & reconstructed & reconstructed & injected \\
(c) & reconstructed & reconstructed & reconstructed \\
(d) & reconstructed & reconstructed & reconstructed \\
& & + time shift & \\
\hline \hline
\end{tabular}

unmodeled algorithm output: $\xi_{\Delta}(\mathbf{t})$ are the detectors responses, while from the estimation of the source direction $(\theta, \phi, \psi)$ we can calculate both the antenna patterns (described by the vectors $\mathbf{F}_{+, x}$ ) and the relative difference in arrival time between detectors $\left(\Delta_{k}\right)$. The system is easily solvable; in fact, applying Cramer's rule, we obtain

$$
\left\{\begin{array}{l}
h_{+}^{(r)}(t)=\frac{\left(\mathbf{F}_{+} \cdot \xi_{\Delta}(\mathbf{t})\right)\left(\left|\mathbf{F}_{\times}\right|^{2}\right)-\left(\mathbf{F}_{\times} \cdot \xi_{\Delta}(\mathbf{t})\right)\left(\mathbf{F}_{+} \cdot \mathbf{F}_{\times}\right)}{\left|\mathbf{F}_{+}\right|^{2}\left|\mathbf{F}_{\times}\right|^{2}-\left(\mathbf{F}_{+} \cdot \mathbf{F}_{\times}\right)^{2}}, \\
h_{\times}^{(r)}(t)=\frac{\left(\mathbf{F}_{\times} \cdot \xi_{\Delta}(\mathbf{t})\right)\left(\left|\mathbf{F}_{+}\right|^{2}\right)-\left(\mathbf{F}_{+} \cdot \xi_{\Delta}(\mathbf{t})\right)\left(\mathbf{F}_{+} \cdot \mathbf{F}_{\times}\right)}{\left|\mathbf{F}_{+}\right|^{2}\left|\mathbf{F}_{\times}\right|^{2}-\left(\mathbf{F}_{+} \cdot \mathbf{F}_{\times}\right)^{2}} .
\end{array}\right.
$$

Equation (3) shows a degeneracy in the regions of the sky where the denominator of the two equations is near zero. The authors of Ref. [45] proposed some solutions to avoid the matrix degeneracy, but we will not consider such an approach in the present study, since for the tested waveforms the number of cases affected by this deficiency is negligible, and therefore it is not necessary to apply any regulator. ${ }^{1}$

To characterize the performance of the new algorithm, we use the Correlation Factor between the original waveform $h^{(i)}$ and the reconstructed waveform $h^{(r)}$ for each polarization, defined as

$$
C_{+, \times}=\frac{\left(h_{+, \times}^{(r)}, h_{+, \times}^{(i)}\right)}{\sqrt{\left(h_{+, \times}^{(r)}, h_{+, \times}^{(r)}\right)\left(h_{+, \times}^{(i)}, h_{+, \times}^{(i)}\right)}},
$$

where we define with (,) the scalar product between two waveforms: $(a, b)=\int a(t) b(t) d t$. The Correlation Factor varies from -1 (opposite matching) to 1 (perfect matching).

The errors in the polarization patterns are derived from the propagation of errors in the estimated quantities: (1) detector response, (2) sky direction, and (3) polarization angle. It is fundamental to characterize the relative propagation of the errors to the polarization reconstruction, so we can understand which one of the just mentioned variables is predominant among the others. We can disentangle the various contributions by solving Eq. (3) in various situations, i.e., by inserting iteratively the real parameters or

\footnotetext{
${ }^{1}$ For $95 \%$ of the sky the value of the denominator is greater than 0.01 .
}

the reconstructed ones. We consider three different cases: (a) reconstructed detector response with true sky location and true polarization angle, (b) reconstructed detector response and sky location with true polarization angle, and (c) reconstructed detector response, sky location, and polarization angle (see Table I).

We should recall that in this paper we are interested in any distortion between the original and reconstructed waveforms; this is why we define the Correlation Factor, which is a suitable quantity for this problem. However, its value can decrease due to a possible time difference between the signal and the reconstructed waveform. An example of this effect is shown in Fig. 1, where we display the values of $C$ when the reconstructed waveform is exactly the original one with different time shifts. We can see that according to the applied time shifts, the values of $C$ vary in the entire possible range of $[-1,1]$. This tells us that we should disentangle the distortions of the signal with respect to any time shifts in the calculation of the Correlation Factor.

We expect a similar behavior in the cases (b) and (c). In fact, case (b) includes a time shift (introduced by the different values of $\Delta_{k}$ ) between the original and reconstructed waveforms. In addition to case (b), case (c) introduces the rotation of the waveform frame $\left[h_{+}^{\prime}=h_{+} \cos (\psi)+h_{\times} \sin (\psi)\right]$ which, especially for sinusoidal waveforms (see Fig. 1), is equivalent to a shift in time. Hence, in case (c) we have two time shifts to take into account: one that is the same as that in case (b), and another that is due to the difference between the original and reconstructed polarization angles The interesting challenge is, do we have a distortion in the signal when we estimate


FIG. 1. Time-shift effect on the Correlation Factor due to rigid shifts of a sinusoid with a frequency of $153 \mathrm{~Hz}$ modulated by a Gaussian envelope. In the top row, the black curves represent the original waveform, while the red/green (left/right) curve is the same waveform after a time shift. In the bottom panel, we show the values of $C$ calculated by applying a time shift ( $x$ axis) to the original waveform. The red and green points refer to the examples in the top row. 
the wrong sky position? Or, is an effect due to a rigid shift as in Fig. 1? To answer the question, we include the case (d), in which we reconstruct the detector response, the sky localization, and also the polarization angle [as in case (c)] and then we add a time shift on $h_{+}$and $h_{\times}$. The applied time shift is the maximum value of the cross-correlation function of time in which we calculate the cross-correlation between the injected and reconstructed polarization patterns. Hence, we estimate the possible shift among the two waveforms and we calculate the $C$ value after correcting the reconstructed polarizations with this time shift. In this way we can focus only on possible signal distortions.

\section{MONTE CARLO SIMULATIONS}

As a starting point for our tool, we take the results obtained by the GW transient signal algorithm in use by the LIGO and Virgo Collaborations called coherent Waveburst $[22,23]$. It is an algorithm to measure energy excesses over the detector noise in the time-frequency domain and combine these excesses coherently among the various detectors. This is performed by introducing a maximum likelihood approach to define the ratio of the probability of having a signal in the data over the probability of having only noise. This does not need a particular assumption on the expected waveform, making it open to a wide class of transient signals.

The algorithm has recently improved in preparation for the advanced detector era. The main improvement concerns a new method for the estimation of the event parameters which considers assumptions on the polarization state (circular, linear, elliptical, etc.) [23]. This improvement is particularly suited for this work because, despite the fact that it does not directly calculate directly the two plus and cross polarizations, it is implicitly connected to them through the calculation of the polarization state. The algorithm performances on sky localization and detector response were reported in the previous results $[43,44]$.

For this work we contemplate a network composed of three interferometers: the two LIGO detectors (L1 in Livingston and $\mathrm{H} 1$ in Hanford) and the Virgo detector (Cascina, Italy), with simulated Gaussian detector noise considering the amplitude spectral density at the design sensitivity $[46,47]$. Even though most of the noise background in detectors is Gaussian, random instrumental artifacts can make the background far from Gaussian $[7,35,48]$. The use of Gaussian noise is just the starting point to verify the performance of this new tool. We expect that glitches would affect only the detection confidence and not the reconstruction of the waveforms, as explained in the case of the sky localization [44]. We will check the real behavior of noise when we handle the data analysis of the three advanced detectors.

We inject the so-called sine-Gaussian and WhiteNoiseBurst waveforms, which are among the standard tested waveforms for burst searches, representing the limiting case for the most simple and complicated morphologies, respectively. The former are defined as follows:

$$
\begin{aligned}
& h_{+}(t)=h \frac{1+\cos ^{2}(l)}{2} \sin \left(2 \pi t f_{0}\right) \exp \left(-t^{2} / \tau^{2}\right) \\
& h_{\times}(t)=h \cos (l) \cos \left(2 \pi t f_{0}\right) \exp \left(-t^{2} / \tau^{2}\right)
\end{aligned}
$$

where $f_{0}$ is the central frequency, $\tau$ is related to the waveform quality factor $Q=\sqrt{2} \pi f_{0} \tau$, and the inclination angle $\imath$ is uniformly distributed. For this study we take into account three quality factors $Q=3,9,100$ and three central frequencies $f_{0}=235,554,1053 \mathrm{~Hz}$, with source coordinates uniformly distributed in the sky. WhiteNoiseBursts are frequency-band-limited white noise with a time Gaussian envelope. They have no particular polarization, while sineGaussians have elliptical polarization. To distinguish the various sources of error, we compute the Correlation Factor [Eq. (4)] for the different cases (a), (b), (c), and (d). To characterize the algorithm's performance, we inject uniformly in the sky at discrete values of the network signal-tonoise ratio (SNR) $(10,12,15,20,25,30$, and 35), where the network SNR is defined as the square sum of the ratio of the reconstructed waveform in the frequency domain $\left(\tilde{h}_{+}, \tilde{h}_{\times}\right)$ and the amplitude spectral density $S_{k}(f)$ of each detector $k: \mathrm{SNR}^{2}=\sum_{k} \int \frac{\tilde{h}_{+}^{2}+\tilde{h}_{\times}^{2}}{S_{k}(f)} d f$.

\section{RESULTS}

The aim of the new algorithm is the reconstruction of both polarizations $(+, \times)$ starting from the results (detector responses, sky localization) of a standard GW transient algorithm. We started the study by disentangling the contribution of detector response and sky localization on the estimation of the polarizations. In Fig. 2 we show an example of the $C_{+}$distribution [Eq. (4)] for an elliptical sine-Gaussian centered at $235 \mathrm{~Hz}$ and $Q=9$ for all of the injected SNR. In case (a), where we have reconstructed only the detector response, the distribution of the Correlation Factor is near 1, confirming that the detector response estimation is in agreement with the expected waveforms for the complete set of SNRs. In case (b), where we reconstruct both the detector response and the sky positions, a new population appears, centered at 0 . However, for case (c), where in addition to the information in case (b) we also reconstruct the polarization angle, there are three main populations centered at $-1,0,1$, respectively. The appearance of these new peaks in cases (b) and (c) can be due to two different effects: signal distortion and/ or time shift. As we have shown in Fig. 1, introducing time shifts lowers the value of the Correlation Factor. When we look at case (d) (where we avoid a possible time shift by bringing the (c) waveform back to the right time), the only remaining distribution is around 1 , similar to what happens in case (a). This answers the question we posed in the previous section: does a wrong sky localization create 



FIG. 2. Correlation Factor of the plus polarization for injections of a sine-Gaussian with a central frequency of $253 \mathrm{~Hz}$ and $Q=9$ uniformly distributed in the sky and for different values of SNR. From left to right, and top to bottom: case (a), (b), (c), and (d).

distortion of the original signal? Results show that the main effect of estimating the wrong sky position is the introduction of a time shift between the estimated polarization and the original one, but such distortions on the waveform shape are negligible. The fact that for case (b) we do not have so many values of the Correlation Factor less than 0 means that the errors from the sky localization cause a time shift to the reconstructed waveform that is not bigger than a certain value. Thinking in terms of phase shift, it is not bigger than a 90 degrees shift. $^{2}$ To confirm this last sentence, we compare the results related to case (b) considering different injection SNRs (Fig. 3) and a sine-Gaussian with different frequencies and the same SNR (Fig. 4).

Figure 3, where we reported three different cases ( $\mathrm{SNR}=12,20$, and 35), shows that the widths of the peaks are not different in the three cases. This verifies that the peak at 0 is not related to the SNR, but it gives a hint that it could be related to the sample rate. However, the fact that the height of the peaks at 0 and at 1 changes for different SNRs tells us that the sky reconstruction is better for high SNR (as expected). The higher the SNR, the smaller the number of events affected by a wrong sky

\footnotetext{
${ }^{2}$ For sinusoidal waveforms, a generic time shift is equivalent to a phase shift.
}

localization, which produces a time shift between the injected and reconstructed waveform polarizations, resulting in low values of the Correlation Factor.

In Fig. 4 we show the performances for waveforms at central frequencies of 554 and $1053 \mathrm{~Hz}$ and compared these with the one at $253 \mathrm{~Hz}$ already reported in Fig. 2. We can see that the distribution of the results is slightly different; in particular, when the frequency increases, the distribution is no longer exhibits the peak at 0 , but the values of the Correlation Factor are more spread out in the possible range $[-1,1]$. The reason is that for this work we adopted the standard sine-Gaussian definition [7,35], which groups waveforms with the same quality factor $Q=\sqrt{2} \pi f_{0} \tau$ into the same set [see Eq. (5)]. The quantity $Q$ is mainly related to the number of cycles that characterize the sine-Gaussian. Therefore, in the same set, given that we have equal cycles but different frequencies, the time duration of the waveforms is inversely proportional to the frequency. Increasing the number of cycles allows the possibility to explore more phase shifts between the injected and the reconstructed waveforms, when we apply a generic time shift between the two waveforms. The time shift between injected and reconstructed waveforms depends only on the sky location and-even if the reconstructed sky localization is better when the frequency increases $[43,49]$ — such improvement 

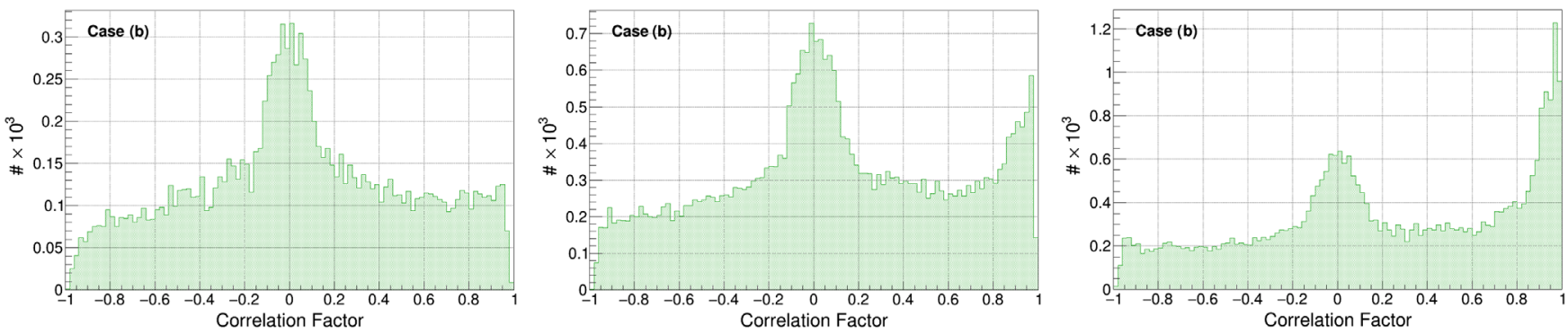

FIG. 3. Correlation Factor of the plus polarization for case (b) for injections of a sine-Gaussian with a central frequency of $253 \mathrm{~Hz}$ and $Q=9$ uniformly distributed in the sky and selected values of SNR. From left to right: SNR = 12, $20,35$.
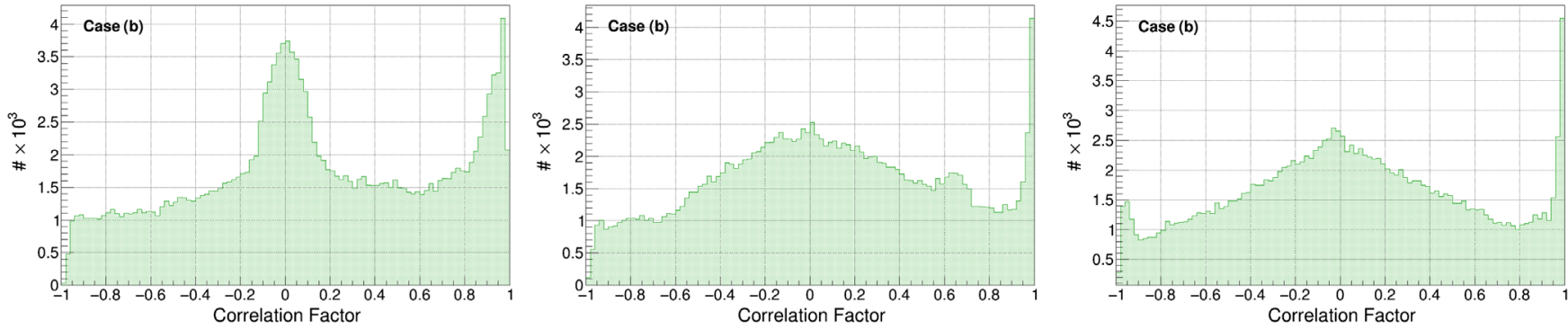

FIG. 4. Correlation Factor of the plus polarization for case (b) for injections of a sine-Gaussian with $Q=9$ uniformly distributed in the sky and selected central frequencies. From left to right, the central frequencies are 235, 554, and $1053 \mathrm{~Hz}$.

is not enough to contrast the effect of having a different number of cycles coming from waveforms at higher frequencies. Increasing the number of cycles allows the possibility to explore more phase shifts between the injected and the reconstructed waveforms, which shapes the distribution of values of the Correlation Factor.

However, for case (c) the reconstructed polarization angle can differ from the original one in the complete range between 0 and 360 degrees, and this is the reason for the peak at -1 . The accumulation point at -1 is always present, for each SNR and frequency, as we can see in Fig. 5. To confirm this we tried the case of injected sky position and reconstructed polarization angle [we call this case (e)], and we show the result for the sine-Gaussian at a central frequency of $235 \mathrm{~Hz}$ and $Q=9$ in Fig. 6 (but for other waveforms, results are similar). Figure 6 shows that the peak at -1 is still present, validating the fact that it is related to the wrong estimation of the polarization angle (moreover, the peak at 0 has disappeared).

In Fig. 7(a) we estimate, from the distribution of Fig. 2, the median value. The shaded regions represent the 30 $70 \%$ percentile of the Correlation Factor. In Fig. 7(b) we have the same results for a WhiteNoiseBurst. We see that for each case [from (a) to (d)] a waveform like a sineGaussian shows in general a median nearer to the optimal value of 1 than a waveform like a WhiteNoiseBurst. These waveforms show a time-frequency representation which involves a greater number of pixels. Having more pixels naturally increases the noise contribution to the waveform reconstruction. This easily explains the reason why performances for a sine-Gaussian are better than those for a WhiteNoiseBurst, given that it is possible to characterize the former with less time-frequency pixels. Indeed, for the sine-Gaussian case, results are independent of the tested


FIG. 5. Correlation Factor of the plus polarization for case (c) for injections of a sine-Gaussian with $Q=9$ uniformly distributed in the sky and selected central frequencies. From left to right, the central frequencies are 235, 554, and $1053 \mathrm{~Hz}$. 




FIG. 6. Correlation Factor of the plus polarization for case (c) for injections of a sine-Gaussian with a central frequency of $253 \mathrm{~Hz}$ and $Q=9$ uniformly distributed in the sky and all of the tested SNRs.

SNR. Since a decrease in the SNR values produces a contamination of the noise in the performances, we chose a network SNR $=10$ (detector SNR $\approx 10 / \sqrt{3}<6$ ) as a reasonable threshold for a candidate event, as explained and done before for searches in Ref. [20].

\section{A. Transient signals}

In this paper we are interested in the reconstruction of the waveform shape with the aim to estimate the characteristics of the generating source from the waveform itself. Given this purpose, we want to focus on eventual signal distortions in the reconstructed polarization patterns. Since in a real analysis we do not know the true quantities, we should focus on case (c). However, as already discussed and shown in Fig. 2, for this case the Correlation Factor $C$ describes the effects of both signal distortion and time shift. Hence, in the following we consider the case (d) because it is connected only to the distortion and not to any eventual time shift applied to the signal.

In Fig. 8(a) we show the comparison of a sine-Gaussian with the same $Q=9$ but at different central frequencies. We can see that performances are better for the plus polarization $(+)$. This is probably because for most of the injection the $(+)$ polarization has higher energy than the cross one $(x)$. Indeed, there are negligible differences for the $(x)$ polarization among the three frequencies, but for the $(+)$ polarization we can see that performances are better for lower frequencies, probably due to the fact that these oscillations are less separated in time. This is also confirmed in Fig. 8(b) where we show a comparison with a sine-Gaussian with the same central frequency but different $Q$. We see that when $Q$ increases, performances are better, because the waveform's bandwidth is narrow and the timefrequency representation involves less pixels.

Similar results for a WhiteNoiseBurst are shown in Fig. 8(c), where the performances are better for lower frequencies. Moreover, for these waveforms the performances



(a) SineGaussian with central frequency of $253 \mathrm{~Hz}$ and $\mathrm{Q}=9$

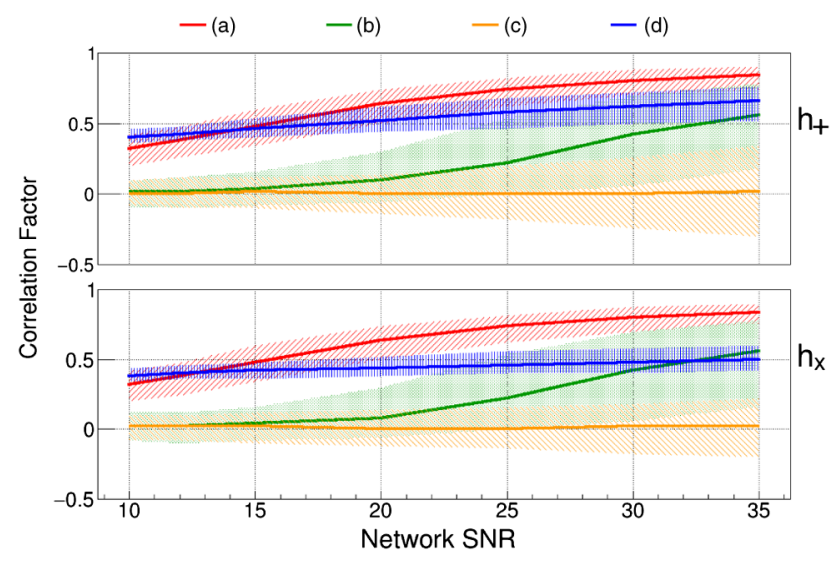

(b) WhiteNoiseBurst with frequency range $(250,350) \mathrm{Hz}$ and duration of $100 \mathrm{~ms}$.



(c) Binary black holes coalescence with single component masses between 15 and 25 solar masses

FIG. 7. Median (lines) and 30-70\% percentile (colored regions) of the Correlation Factor for the plus (top) and cross (bottom) polarization for injections uniformly distributed in the sky as a function of the injected SNR. Colors refer to cases (a) (red), (b) (green), (c) (orange), and (d) (blue).

are slightly worse with respect to a sine-Gaussian. Indeed, the WhiteNoiseBurst signals are more widespread in the time-frequency domain than the sine-Gaussian, which makes it more difficult to accurately reconstruct the 


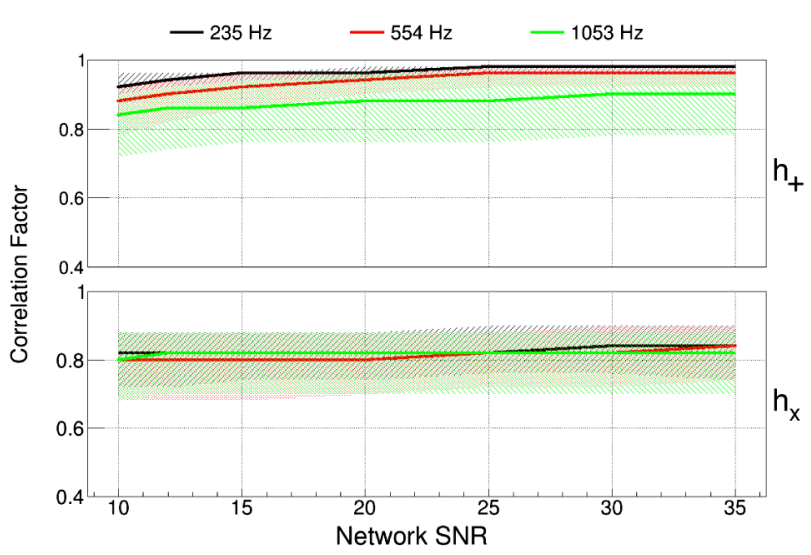

(a) SineGaussian with $\mathrm{Q}=9$ and central frequency at $235 \mathrm{~Hz}$ (black), $554 \mathrm{~Hz}$ (red), $1053 \mathrm{~Hz}$ (green).

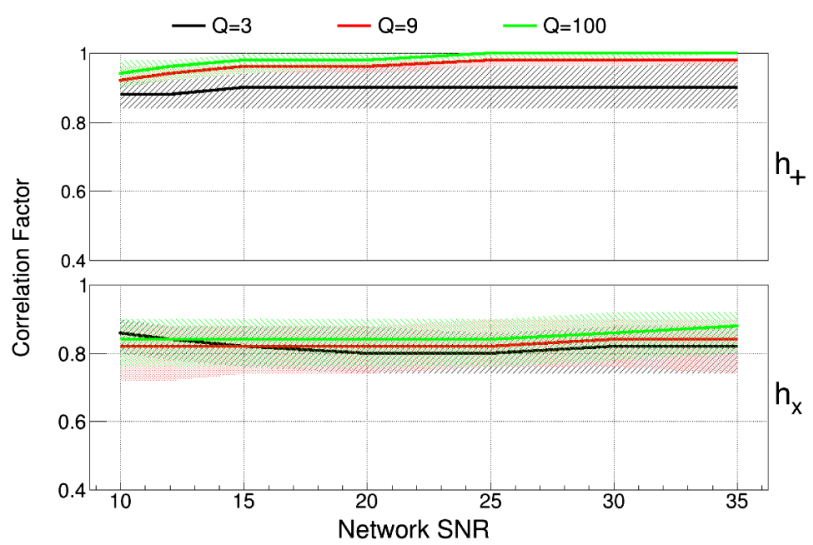

(b) SineGaussian with central frequency $=235 \mathrm{~Hz}$ and $\mathrm{Q}=3$ (black), $\mathrm{Q}=9$ (red), $\mathrm{Q}=100$ (green).

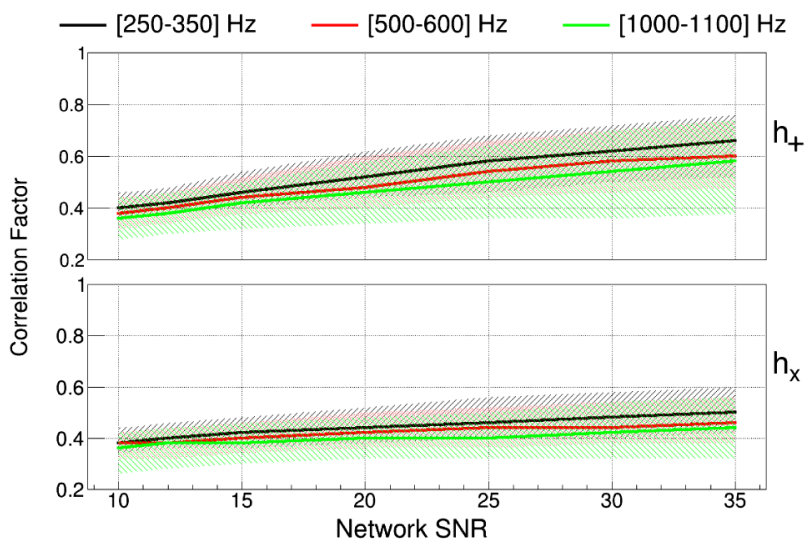

(c) WhiteNoiseBurst with bandwidth $(250,350) \mathrm{Hz}$ (black), $(500,600) \mathrm{Hz}$ (red) and $(1000,1100) \mathrm{Hz}$ (green).

FIG. 8. Median (lines) and 30-70\% percentile (colored regions) of the Correlation Factor for the plus (top) and cross (bottom) polarization for different signals for the (d) case. The $y$ axis shows the Correlation Factor value for which the 50\% (lines) and 30-70\% percentiles of the recovered waveforms have a bigger cross correlation.

complete waveform. As already shown [Fig. 7(b)], for these waveforms the contribution to the errors coming from the detector response estimation is more important than the sine-Gaussian.

\section{B. Compact binary coalescence}

For completeness, we also test the algorithm on a set of signals coming from the coalescence of binary black hole systems. The first detections of gravitational waves were generated by the merger of two black holes, which are the most cataclysmic events in nature. In such systems two black holes combine to form a single one, emitting a strong gravitational wave. In our simulations we consider binary black hole systems with single masses between 15 and 25 solar masses and uniform spin distribution between 0 and 0.9 . These are the same waveforms used in Ref. [44], where the Coherent WaveBurst performances on sky localization have already been reported. Waveforms are injected up to $4 \mathrm{Gpc}$, using a uniform distribution in volume. We do consider any cosmological evolution, so in the previous values we are referring to masses in the source frames and luminosity distance. The reconstructed events are collected in bins of network SNR, and each bin has a width equal to 2 . To be homogeneous with the results of the other waveforms we consider the SNR range 8-38. Results from these tests are shown in Fig. 7(c). The results are similar to the case of WhiteNoiseBursts in Fig. 7(b). Indeed the signals belonging to these two classes involve a greater number of pixels in the time-frequency domain. This increases the noise contribution to the waveform estimation, which makes it more difficult to accurately reconstruct the complete waveform.

\section{CONCLUSION}

We have tested a new algorithm for estimating the inverse solution of gravitational wave transient signals from the information given by a nontemplate search, and reconstructed the original waveform polarization. This approach is the first attempt to use and verify the accuracy of the tool. Assuming Gaussian noise and that the detectors operate at the design sensitivity, our results show a reliable reconstruction of both $(+)$ and $(x)$ polarizations, even for low injected values of SNR.

Through the disentanglement of the various error contributions, we have shown that the main effect is due to the detector response. In fact, the correction caused by the sky position is mainly a time shift of the signal proper time, but no significant distortion appears in the original waveform, as seen in Fig. 2.

We are working to further improve and refine the reconstruction by reducing the contribution of the noise in the detector response estimation. In future works, we hope to verify whether real detector noise gives comparable results and to study the effect of the matrix deficiency, for instance, by applying the approach proposed in Ref. [45]. This tool does not rely on a specific pipeline; hence, it can be applied to any algorithm that provides information about the detector response and sky location. It would be 
interesting to apply this approach to events detected by Advanced LIGO and Virgo in the future observational runs. For events detected with template searches, it would be straightforward to compare the polarizations obtained with this method with the ones given by the template. This would allow to check the performances of this approach with the best matching, but it could also give hints on possible variations of the real signal from the template itself. For events detected with unmodeled algorithms, reconstructing the polarizations would be the first step to understanding the generating process, other than the astrophysical progenitor.

\section{ACKNOWLEDGMENTS}

The authors gratefully acknowledge the support of the research by the Italian Istituto Nazionale di Fisica Nucleare and the Max-Planck-Society, and the State of Niedersachsen/Germany. They also gratefully acknowledge the support of L'Oréal Italia For Women in Science.
[1] A. Einstein, Näherungsweise Integration der Feldgleichungen der Gravitation, Sitzungsber. K. Preuss. Akad. Wiss. 1, 688 (1916).

[2] A. Einstein, Über Gravitationswellen, Sitzungsber. K. Preuss. Akad. Wiss. 1, 154 (1918).

[3] R. A. Hulse and J. H. Taylor, Discovery of a pulsar in a binary system, Astrophys. J. 195, L51 (1975).

[4] H. Taylor and J. M. Weisberg, A new test of general relativity - Gravitational radiation and the binary pulsar PSR 1913+16, Astrophys. J. 253, 908 (1982).

[5] J. Aasi et al., Characterization of the LIGO detectors during their sixth science run, Classical Quantum Gravity 32, 115012 (2015).

[6] T. Accadia et al., Virgo: a laser interferometer to detect gravitational waves, J. Instrum. 7, P03012 (2012).

[7] J. Abadie et al., All-sky search for gravitational-wave bursts in the second joint LIGO-Virgo run, Phys. Rev. D 85, 122007 (2012).

[8] J. Abadie et al., Search for gravitational waves from low mass compact binary coalescence in LIGO's sixth science run and Virgo's science runs 2 and 3, Phys. Rev. D 85, 082002 (2012).

[9] J. Abadie et al., First all-sky search for continuous gravitational waves from unknown sources in binary systems, Phys. Rev. D 90, 062010 (2014).

[10] J. Aasi et al., Improved Upper Limits on the Stochastic Gravitational-Wave Background from 2009-2010 LIGO and Virgo Data, Phys. Rev. Lett. 113, 231101 (2014).

[11] H. Grote et al., The GEO 600 status,Classical Quantum Gravity 27, 084003 (2010).

[12] J. Abadie et al., Advanced LIGO, Classical Quantum Gravity 32, 074001 (2015).

[13] F. Acernese et al., Advanced Virgo: a second-generation interferometric gravitational wave detector, Classical Quantum Gravity 32, 024001 (2015).

[14] G. M. Harry, Advanced LIGO: the next generation of gravitational wave detectors, Classical Quantum Gravity 27, 084006 (2010).

[15] Virgo Collaboration, Advanced Virgo technical design report, 2012, VIR-0128A-12.

[16] Y. Aso, Y. Michimura, K. Somiya, M. Ando, O. Miyakawa, T. Sekiguchi, D. Tatsumi, and H. Yamamoto, Interferometer design of the KAGRA gravitational wave detector, Phys. Rev. D 88, 043007 (2013).

[17] K. Somiya, Detector configuration of KAGRA-the Japanese cryogenic gravitational-wave detector, Classical Quantum Gravity 29, 124007 (2012).

[18] B. Iyer et al., LIGO-India, Proposal of the Consortium for Indian Initiative in Gravitational-wave Observations (IndIGO), 2011, https://dcc.ligo.org/LIGO-M1100296/public.

[19] B. P. Abbott et al., Observation of Gravitational Waves from a Binary Black Hole Merger, Phys. Rev. Lett. 116, 061102 (2016).

[20] B. P. Abbott et al., Observing gravitational-wave transient GW150914 with minimal assumptions, Phys. Rev. D 93, 122004 (2016).

[21] B. P. Abbott et al., GW150914: First results from the search for binary black hole coalescence with Advanced LIGO, Phys. Rev. D 93, 122003 (2016).

[22] S. Klimenko, I. Yakushin, A. Mercer, and G. Mitselmakher, A coherent method for detection of gravitational wave bursts, Classical Quantum Gravity 25, 114029 (2008).

[23] S. Klimenko et al., Method for detection and reconstruction of gravitational wave transients with networks of advanced detectors, Phys. Rev. D 93, 042004 (2016).

[24] B. P. Abbott et al., Tests of General Relativity with GW150914, Phys. Rev. Lett. 116, 221101 (2016).

[25] B. P. Abbott et al., Properties of the Binary Black Hole Merger GW150914, Phys. Rev. Lett. 116, 241102 (2016).

[26] B. P. Abbott et al., GW151226: Observation of Gravitational Waves from a 22-Solar-Mass Binary Black Hole Coalescence, Phys. Rev. Lett. 116, 241103 (2016).

[27] B. P. Abbott et al., GW170104: Observation of a 50-SolarMass Binary Black Hole Coalescence at Redshift 0.2, Phys. Rev. Lett. 118, 221101 (2017).

[28] B. P. Abbott et al., GW170608: Observation of a 19-solarmass Binary Black Hole Coalescence, Astrophys. J. 851, L35 (2017).

[29] B. P. Abbott et al., Binary Black Hole Mergers in the first Advanced LIGO Observing Run, Phys. Rev. X 6, 041015 (2016).

[30] B. P. Abbott et al., GW170814: A Three-Detector Observation of Gravitational Waves from a Binary Black Hole Coalescence, Phys. Rev. Lett. 119, 141101 (2017). 
[31] B. P. Abbott et al., GW170817: Observation of Gravitational Waves from a Binary Neutron Star Inspiral, Phys. Rev. Lett. 119, 161101 (2017).

[32] B. P. Abbott et al., Gravitational Waves and Gamma-rays from a Binary Neutron Star Merger: GW170817 and GRB 170817A, Astrophys. J. 848, L13 (2017).

[33] B. P. Abbott et al., A gravitational-wave standard siren measurement of the Hubble constant, Nature (London) $\mathbf{5 5 1}$, 85 (2017).

[34] B. P. Abbott et al., Astrophysical Implications of the Binary Black-Hole Merger GW150914, Astrophys. J. 818, L22 (2016).

[35] J. Abadie et al., All-sky search for gravitational-wave bursts in the first joint LIGO-GEO-Virgo run, Phys. Rev. D 81, 102001 (2010).

[36] S. Chatterji, A. Lazzarini, L. Stein, P. Sutton, A. Searle, and M. Tinto, Coherent network analysis technique for discriminating gravitational-wave bursts from instrumental noise, Phys. Rev. D 74, 082005 (2006).

[37] W. G. Anderson, P. R. Brady, J. D. E. Creighton, and E. E. Flanagan, An Excess power statistic for detection of burst sources of gravitational radiation, Phys. Rev. D 63, 042003 (2001).

[38] L. Wen and B. Schutz, Coherent network detection of gravitational waves: The Redundancy veto, Classical Quantum Gravity 22, S1321 (2005).

[39] L. Cadonati, Coherent waveform consistency test for LIGO burst candidates, Classical Quantum Gravity 21, S1695 (2004).

[40] N. Cornish and T. Littenberg, BayesWave: Bayesian Inference for Gravitational Wave Bursts and Instrument Glitches, Classical Quantum Gravity 32, 135012 (2015).
[41] B. S. Sathyaprakash and B. F. Schutz, Physics, astrophysics and cosmology with gravitational waves, Living Rev. Relativity 12, 2 (2009).

[42] S. Klimenko, S. Mohanty, M. Rakhmanov, and G. Mitselmakher, Constraint likelihood analysis for a network of gravitational wave detectors, Phys. Rev. D 72, 122002 (2005).

[43] S. Klimenko, G. Vedovato, M. Drago, G. Mazzolo, G. Mitselmakher, C. Pankow, G. Prodi, V. Re, F. Salemi, and I. Yakushin, Localization of gravitational wave sources with networks of advanced detectors, Phys. Rev. D 83, 102001 (2011).

[44] R. Essick, S. Vitale, E. Katsavounidis, G. Vedovato, and S. Klimenko, Localization of short duration gravitationalwave transients with the early advanced LIGO and Virgo detectors, Astrophys. J. 800, 81 (2015).

[45] M. Rakhmanov, Rank deficiency and Tikhonov regularization in the inverse problem for gravitational-wave bursts, Classical Quantum Gravity 23, S673 (2006).

[46] Advanced LIGO anticipated sensitivity curves, https://dcc .ligo.org/LIGO-T0900288/public.

[47] Advanced Virgo Baseline Design, https://tds.ego-gw.it/q1/? $\mathrm{c}=6589$.

[48] B. P. Abbott et al., All-sky search for short gravitationalwave bursts in the first Advanced LIGO run, Phys. Rev. D 95, 042003 (2017).

[49] S. Fairhust, Source localization with an advanced gravitational wave detector network, Classical Quantum Gravity 28, 105021 (2011). 